\title{
Asymptomatic Abdominal Aortic Aneurysms Show Histological Signs of Progression: A Quantitative Histochemical Analysis
}

\author{
Lada Eberlováa, ${ }^{a}$ Zbyněk Tonar ${ }^{a, f}$ Kirsti Witter ${ }^{g}$ Věra Kř́žkováa \\ Lukáš Nedorost $^{\mathrm{a}}$ Marie Korabečnác ${ }^{\text {Pavel Tolinger }}{ }^{\mathrm{a}}$ Jitka Kočováa $^{\mathrm{a}}$

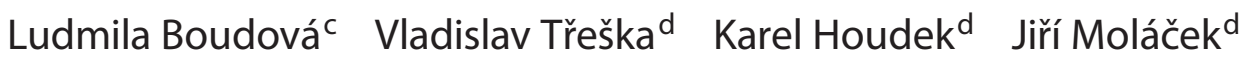 \\ Jindra Vrzalováe Martin Peštae ${ }^{e}$ Ondřej Topolčan ${ }^{\mathrm{e}}$ Jiří Valenta $^{\mathrm{b}}$
}

\begin{abstract}
Departments of a Histology and Embryology and ${ }^{b}$ Anatomy, Faculty of Medicine in Pilsen, Departments of cPathology, d Surgery and eNuclear Medicine and Central Radioisotopic Laboratory, Charles University in Prague, University Hospital in Pilsen, and ${ }^{\mathrm{f} E u r o p e a n ~ C e n t r e ~ o f ~ E x c e l l e n c e ~ N T I S ~-~ N e w ~ T e c h n o l o g i e s ~ f o r ~ I n f o r m a t i o n ~ S o c i e t y, ~}$ Faculty of Applied Sciences, University of West Bohemia, Pilsen, Czech Republic; ${ }^{9}$ Institute of Anatomy, Histology and Embryology, Department of Pathobiology, University of Veterinary Medicine Vienna, Vienna, Austria
\end{abstract}

\section{Key Words}

Abdominal aortic aneurysm $\cdot$ Histology

Immunohistochemistry $\cdot$ Stereology $\cdot$ Multiplex

immunoanalysis

\begin{abstract}
Objective: Abdominal aortic aneurysm (AAA) is a serious disease due to its covert nature, relatively high prevalence and fatal prognosis in the case of rupture. To obtain new insights into AAA pathogenesis, we examined the relationships between histopathology, multiplex in vitro immunoassay data, diameter and symptomatology. Methods: In a prospective, non-randomised study, we evaluated samples from 6 normal infrarenal aortae and 65 AAA patients (65 walls, 55 thrombi). The AAA patients were either asymptomatic ( $\mathrm{n}=$ $44)$, symptomatic $(n=7)$ or with ruptured AAA $(n=14)$. The AAA diameter was classified as small $(\angle 5 \mathrm{~cm}, \mathrm{n}=18)$, medium $(5-7 \mathrm{~cm}, \mathrm{n}=26)$ and large $(>7 \mathrm{~cm}, \mathrm{n}=21)$. We quantified the histopathology of the AAA wall and the adjacent thrombus. We assessed the expression of proteins in the same samples. Results: Asymptomatic AAAs had walls with more abundant
\end{abstract}

\section{KARGER}

Fax +4161306 1234 E-Mail karger@karger.ch www.karger.com
(C) 2012 S. Karger AG, Basel

1015-2008/13/0801-0011\$38.00/0

Accessible online at:

www.karger.com/pat inflammatory infiltrates, lower amounts of PAI-1, a higher number of tPA-positive elements, a tendency towards decreased collagen content, whereas the adjacent thrombi had a greater concentration of VCAM- 1 and MMP- 2 when compared with symptomatic AAAs. Compared with the aneurysmatic aorta, the normal aorta contained less collagen and more elastin, actin, desmin and PAI-1-positive elements; in addition, it was more vascular. Medium-sized AAAs were the most actin and vimentin rich, and large AAAs were the most vascular. Conclusion: Our results show that asymptomatic AAA walls often have more potentially deleterious histopathological alterations than symptomatic AAA walls. This result indicates that a progression from an asymptomatic AAA to rupture can be expected and screening patients who are at risk of rupture could be beneficial.

Copyright $\odot 2012$ S. Karger AG, Basel

Abdominal aortic aneurysm (AAA) appears to be a specific form of atherothrombosis [1] in which progressive degradation leads to dilatation and rupture. However, the role of atherosclerosis is subject to debate [2]. Aneurysms 
are defined as focal, irreversible dilatations of all layers of a vessel wall with a diameter of at least one and a half times larger than the normal diameter of the respective vessel. In the abdominal aorta, an aneurysm is most frequently found in the infrarenal segment, and a diameter larger than $3.0 \mathrm{~cm}$ is considered to be an aneurysm [3].

AAA is a grave disease, most common in men aged 65 years and older. The majority of AAAs are asymptomatic [4]. The most common symptom of AAA is lumbago, whilst, more rarely, abdominal pulsation can be observed. About a quarter of untreated AAAs end with rupture [5], which has a mortality rate of $70-80 \%$ [6]. The risk of rupture increases with an increasing diameter of the aneurysm [7]. The epidemiological profile of AAA seems to be changing. Studies reported an AAA prevalence in the range of $4-7 \%$ for men aged 65 years or more $[8,9]$ and increasing rates in incidence [10] and mortality [11]. Recent studies have shown a decrease of AAA prevalence to less than $2 \%$ in men aged $\geq 65$ years [12] and there is evidence that the incidence and mortality from AAA is also declining [13]. These results may reflect the positive effects of reduced exposure to risk factors.

The pathogenesis of AAA is multifactorial and the precise causes and development of the disease are not known. Degeneration of the vessel wall and subsequent development of aneurysms involves several interconnected processes, including chronic inflammation [14] accompanied by neovascularisation [15], progressive destruction and loss of elastin [16], vascular smooth muscle cell (VSMC) phenotypic modulation [17] and VSMC apoptosis [18]. Inflammatory infiltrates consist mainly of macrophages and $\mathrm{T}$ and $\mathrm{B}$ lymphocytes $[14,19]$. Newly recruited and residential inflammatory cells produce cytokines and chemokines [20] that amplify the immune response and induce the expression of proteases such as serine proteases, plasminogen activators and metalloproteinases $[21,22]$. Intraluminal thrombus also participates in aneurysmal dilatation $[23,24]$ and represents a significant source of oxidative and proteolytic enzymes that contribute to wall injury $[25,26]$. However, the presence of intraluminal thrombi strengthens the AAA wall and positively affects the redistribution and reduction of the wall stress [27]. Current knowledge on intraluminal thrombi in AAA is summarized by Yoshimura et al. [28].

Discovery of an applicable medication that would effectively reduce the progression of small aneurysms requires a detailed understanding of AAA pathogenesis and strongly supports arguments for establishing a screening protocol for AAAs [29]. The aim of our study was to contribute to the knowledge of AAA progression by quantitatively analysing histopathological changes in the aortic wall and the associated thrombus in relation to the severity of the disease (i.e. healthy aorta vs. aneurysm, symptomatology, and AAA size). Particular attention was paid to signs of inflammation, including vascularisation, shifts in the VSMC phenotype, and remodelling of the extracellular matrix of the aortic wall (i.e. components that may influence the mechanical properties of the vessel wall).

\section{Materials and Methods}

Patients and Tissue Samples

We evaluated samples from the infrarenal AAA wall $(n=65$; 51 men, 14 women) and the intraluminal thrombi of the same patients ( $\mathrm{n}=55$; in 10 patients the thrombus was either too small or not present). Specimens were collected from patients undergoing elective or emergency surgery for atherosclerotic AAAs during the years 2008-2010. The average age of the patients was 73 (5792) years. According to the maximum aortic diameter (measured by CT angiography), AAAs were classified as small $(<5 \mathrm{~cm}, \mathrm{n}=$ $18)$, medium $(5-7 \mathrm{~cm}, \mathrm{n}=26)$ and large $(>7 \mathrm{~cm}, \mathrm{n}=21)$. Most of the cases of AAA $(68 \%)$ were asymptomatic $(n=44)$. Some cases were symptomatic $(n=7)$ or ruptured $(n=14)$. Symptomatic cases were defined as aneurysms that were accompanied by lumbago, stomach ache, aortic palpation sensitivity or peripheral embolisation. We aimed to find the differences between the groups of patients defined based on the size of the aneurysms and symptomatology. Regarding the medications that influence AAA growth [reviewed in 30, 31], 58\% of patients with AAA used statins, $49 \%$ used acetylsalicylic acid, 35\% used beta blockers and 28\% used ACE inhibitors. In the group with small, asymptomatic AAAs $(\mathrm{n}=16), 88 \%$ of the patients were treated with statins and $75 \%$ were treated with acetylsalicylic acid. Normal aortas $(n=6)$ were taken from the infrarenal segment of organ donors with an average age of 47 (28-64) years.

AAA specimens were always excised from the anterior wall of the sac at the maximum diameter. Samples from normal aortas were fixed with buffered formalin for histological and immunohistochemical examination. For in vitro immunoassays, tissues and thrombus pieces taken from the locations described above were chopped into approximately $1.5 \mathrm{~mm}$ cubes, placed into Eppendorf tubes, frozen in liquid nitrogen and stored in the freezer at $-70^{\circ} \mathrm{C}$. The study was approved by the Ethical Committee at the University Hospital and the Faculty of Medicine of Charles University in Pilsen.

\section{Histology and Immunohistochemistry}

Fixed specimens from the aortic wall and thrombi were dehydrated in graded ethanol solutions and embedded in paraffin. Tissue blocks were cut transversally into $3-5-\mu \mathrm{m}$ sections, mounted on Super Frost slides coated with (3-aminopropyl) triethoxysilane (Sigma Aldrich, Vienna, Austria), deparaffinised, rehydrated and processed as follows: two sections per block were stained with haematoxylin-eosin and two sections were stained with Verhoeff's haematoxylin and green trichrome to differenti- 
Table 1. Immunohistochemical details for the histological analysis

\begin{tabular}{|c|c|c|}
\hline Primary antibody & Blocking steps and pretreatment & $\begin{array}{l}\text { Detection of the } \\
\text { immunoreaction }\end{array}$ \\
\hline $\begin{array}{l}\text { Monoclonal Mouse Anti-Human Smooth } \\
\text { Muscle Actin, Clone 1A4 (Dako), 1:400 }\end{array}$ & $\begin{array}{l}0.6 \% \mathrm{H}_{2} \mathrm{O}_{2} \text { in methanol; } 1.5 \% \text { normal goat serum } \\
\text { (Dako) in PBS; no pretreatment }\end{array}$ & $\begin{array}{l}\text { Anti-mouse PowerVision Kit } \\
\text { (Immunovision) }\end{array}$ \\
\hline $\begin{array}{l}\text { Monoclonal Mouse Anti-Human Desmin, } \\
\text { Clone D33 (marker of the smooth muscle } \\
\text { phenotype) (Dako), 1:300 }\end{array}$ & $\begin{array}{l}0.6 \% \mathrm{H}_{2} \mathrm{O}_{2} \text { in methanol; } 1.5 \% \text { normal goat serum } \\
\text { (Dako) in PBS; microwave pretreatment } 3 \times 5 \text { min } \\
\text { in Tris-EDTA pH } 9.0 \text { according to the manufacturer } \\
\text { (Dako) }\end{array}$ & $\begin{array}{l}\text { Anti-mouse PowerVision Kit } \\
\text { (Immunovision) }\end{array}$ \\
\hline $\begin{array}{l}\text { Monoclonal Mouse Anti-Human Vimen- } \\
\text { tin, Clone V9 (marker of the fibroblastic } \\
\text { phenotype; Dako), 1:200 }\end{array}$ & $\begin{array}{l}0.6 \% \mathrm{H}_{2} \mathrm{O}_{2} \text { in methanol; } 1.5 \% \text { normal goat serum } \\
(\mathrm{Dako}) \text { in PBS; microwave pretreatment } 2 \times 5 \mathrm{~min} \\
\text { in } 0.01 \mathrm{M} \text { citrate buffer, } \mathrm{pH} 6.0\end{array}$ & $\begin{array}{l}\text { Anti-mouse PowerVision Kit } \\
\text { (Immunovision) }\end{array}$ \\
\hline $\begin{array}{l}\text { Polyclonal Rabbit Anti-Human Myeloper- } \\
\text { oxidase (granulocyte marker; Fisher), 1:300 }\end{array}$ & $\begin{array}{l}0.6 \% \mathrm{H}_{2} \mathrm{O}_{2} \text { in methanol; } 1.5 \% \text { normal goat serum } \\
\text { (Dako) in PBS; microwave pretreatment } 3 \times 5 \mathrm{~min} \\
\text { in } 0.01 \mathrm{M} \text { citrate buffer, } \mathrm{pH} 6.0\end{array}$ & $\begin{array}{l}\text { Anti-rabbit PowerVision Kit } \\
\text { (Immunovision) }\end{array}$ \\
\hline $\begin{array}{l}\text { Monoclonal Mouse Anti-Macrophage / } \\
\text { L1Protein / Calprotectin, Clone MAC } 387 \\
\text { (macrophage marker; Fisher), 1:200 }\end{array}$ & $\begin{array}{l}0.6 \% \mathrm{H}_{2} \mathrm{O}_{2} \text { in methanol; } 1.5 \% \text { normal goat serum } \\
\text { (Dako) in PBS; digestion with } 1 \mathrm{mg} \text { protease from } \\
\text { Streptomyces griseus (Sigma)/ml PBS for } 15 \text { min at } \\
\text { room temperature }\end{array}$ & $\begin{array}{l}\text { Anti-mouse PowerVision Kit } \\
\text { (Immunovision) }\end{array}$ \\
\hline $\begin{array}{l}\text { Monoclonal Mouse Anti-Human CD31, } \\
\text { Clone JC70A (endothelial marker; Dako), } \\
\text { 1:30 }\end{array}$ & $\begin{array}{l}0.6 \% \mathrm{H}_{2} \mathrm{O}_{2} \text { in methanol; } 1.5 \% \text { normal goat serum } \\
(\mathrm{Dako}) \text { in PBS; microwave pretreatment } 4 \times 5 \mathrm{~min} \\
\text { in } 0.01 \mathrm{M} \text { citrate buffer, } \mathrm{pH} 6.0\end{array}$ & $\begin{array}{l}\text { Anti-mouse PowerVision Kit } \\
\text { (Immunovision) }\end{array}$ \\
\hline $\begin{array}{l}\text { Polyclonal Goat Anti-Human PAI-1 (C-20; } \\
\text { plasminogen activator inhibitor-1; Santa } \\
\text { Cruz), 1:50 }\end{array}$ & $\begin{array}{l}1 \% \mathrm{H}_{2} \mathrm{O}_{2} \text { in } \mathrm{PBS} ; 1.5 \% \text { donkey blocking serum (San- } \\
\text { ta Cruz) in PBS; water bath }\left(95^{\circ} \mathrm{C}\right) \text { pretreatment } 30 \\
\text { min in } 0.01 \mathrm{M} \text { citrate buffer, } \mathrm{pH} 6.0\end{array}$ & $\begin{array}{l}\text { ImmunoCruz } \\
\text { Staining System (Santa Cruz) }\end{array}$ \\
\hline $\begin{array}{l}\text { Polyclonal Goat Anti-Human tPA (C-16; } \\
\text { tissue plasminogen activator; Santa Cruz), } \\
\text { 1:50 }\end{array}$ & $\begin{array}{l}1 \% \mathrm{H}_{2} \mathrm{O}_{2} \text { in } \mathrm{PBS} ; 1.5 \% \text { donkey blocking serum (San- } \\
\text { ta Cruz) in PBS; water bath }\left(95^{\circ} \mathrm{C}\right) \text { pretreatment } 30 \\
\text { min in } 0.01 \mathrm{M} \text { citrate buffer, pH } 6.0\end{array}$ & $\begin{array}{l}\text { ImmunoCruz }{ }^{\mathrm{TM}} \text { goat ABC } \\
\text { Staining System (Santa Cruz) }\end{array}$ \\
\hline $\begin{array}{l}\text { Polyclonal Goat Anti-Human uPA (C-20; } \\
\text { urinary plasminogen activator; Santa Cruz), } \\
\text { 1:50 }\end{array}$ & $\begin{array}{l}1 \% \mathrm{H}_{2} \mathrm{O}_{2} \text { in } \mathrm{PBS} ; 1.5 \% \text { donkey blocking serum (San- } \\
\text { ta Cruz) in PBS; water bath }\left(95^{\circ} \mathrm{C}\right) \text { pretreatment } 30 \\
\text { min in } 0.01 \mathrm{M} \text { citrate buffer, } \mathrm{pH} 6.0\end{array}$ & $\begin{array}{l}\text { ImmunoCruz }{ }^{\mathrm{TM}} \text { goat ABC } \\
\text { Staining System (Santa Cruz) }\end{array}$ \\
\hline
\end{tabular}

Primary antibodies, blocking of endogenous peroxidase activity and non-specific binding of antibodies, pretreatment and detection kits used for immunohistochemistry. PBS = Phosphate-buffered saline. (Dako: DakoCytomation, Glostrup, Denmark; Immunovision: Immunovision Technologies, Daly City, Calif., USA; Fisher: Thermo Fisher Scientific, Fremont, Calif., USA; Sigma: Sigma Aldrich, Vienna, Austria; Santa Cruz: Santa Cruz Biotechnology, Santa Cruz, Calif., USA.)

ate elastic and collagen fibres. Additional sections were processed immunohistochemically. The VSMC phenotype was characterised based on immunopositivity for $\alpha$-smooth muscle actin, desmin and vimentin; with actin and desmin characterising predominantly contractile VSMC, and vimentin the predominantly synthetic phenotype [32]. Immunohistochemistry was used to detect granulocytes, macrophages and regulators of fibrinolysis, including $\mathrm{UPA}, \mathrm{tPA}, \mathrm{PAI}-1$ and vasa vasorum endothelium. A list of the primary antibodies used in this study, including the manufacturer, dilution, pretreatment and appropriate detection systems, is provided in table 1. Endogenous peroxidase activity was blocked in the immunohistochemistry experiments. Tissue sections (except for the detection of fibrinolytic components) were permeabi- lised with chilled acetone $\left(-20^{\circ} \mathrm{C}\right)$ for $10 \mathrm{~min}$. Non-specific binding activity was blocked with serum at room temperature. After the pre-treatments, sections were incubated overnight with primary antibodies at $4{ }^{\circ} \mathrm{C}$. The products of the immunoreactions were detected using commercially available detection systems (table 1). The reactions were visualised with diaminobenzidine (Sigma Aldrich) in $0.03 \% \mathrm{H}_{2} \mathrm{O}_{2}$ in Tris-buffered saline ( $\mathrm{pH}$ 7.4). All of the sections were counterstained with haematoxylin, dehydrated and mounted using a medium soluble in xylene. For negative controls, normal goat serum was used instead of the primary antibodies. Normal tissue with typical morphology in the sections of AAA walls, e.g. aortic lymph nodes for immune cells and smooth muscle cells of small arteries, served as a positive control. 
Table 2. Sampling of histological sections and microscopic image fields for estimating the quantitative parameters of the aortic wall and thrombi

\begin{tabular}{|c|c|c|c|c|}
\hline $\begin{array}{l}\text { Quantitative parameter } \\
\text { (component, ref. space) }\end{array}$ & $\begin{array}{l}\text { Microscope } \\
\text { objective } \\
\text { used }\end{array}$ & $\begin{array}{l}\text { Sections (and } \\
\text { image fields) } \\
\text { sampled per patient }\end{array}$ & $\begin{array}{l}\text { Total area } \\
\text { sampled in each } \\
\text { tissue block, } \mathrm{mm}^{2}\end{array}$ & Number of counting events per patient \\
\hline $\mathrm{A}_{\mathrm{A}}($ elastin, int + med $)$ & $40 \times$ & $1(8)$ & 1.59 & $>200$ intersections with elastin \\
\hline $\mathrm{A}_{\mathrm{A}}($ collagen, int + med $)$ & $20 \times$ & $1(12)$ & 9.52 & $>200$ intersections with collagen \\
\hline $\mathrm{L}_{\mathrm{A}}($ elastin, int + med $)$ & $40 \times$ & $1(8)$ & 1.59 & $>200$ intersections with elastin \\
\hline $\mathrm{A}_{\mathrm{A}}($ actin, int + med $)$ & $40 \times$ & $1(8)$ & 1.59 & $>200$ intersections with actin-positive areas \\
\hline $\mathrm{A}_{\mathrm{A}}($ desmin, int + med $)$ & $40 \times$ & $1(8)$ & 1.59 & $>200$ intersections with desmin-positive areas \\
\hline $\mathrm{A}_{\mathrm{A}}($ vimentin, int + med $)$ & $40 \times$ & $1(8)$ & 1.59 & $>200$ intersections with vimentin-positive areas \\
\hline $\mathrm{Q}_{\mathrm{A}}$ & $10 \times$ & $1(4)$ & 12.69 & $98-253$ profiles of vasa vasorum, 168 on average \\
\hline $\mathrm{A}_{\mathrm{A}}($ granulocytes, wall) & $40 \times$ & $1(8)$ & 1.59 & $>150$ intersections with granulocyte-positive areas \\
\hline $\mathrm{A}_{\mathrm{A}}($ macrophages, wall $)$ & $40 \times$ & $1(8)$ & 1.59 & $>150$ intersections with macrophage-positive areas \\
\hline $\mathrm{A}_{\mathrm{A}}(t P A$, wall $)$ & $40 \times$ & $1(8)$ & 1.59 & $>200$ intersections with tPA-positive components \\
\hline $\mathrm{A}_{\mathrm{A}}(u P A$, wall $)$ & $40 \times$ & $1(8)$ & 1.59 & $>200$ intersections with uPA-positive components \\
\hline $\mathrm{A}_{\mathrm{A}}(P A I-1$, wall $)$ & $40 \times$ & $1(8)$ & 1.59 & $>200$ intersections with PAI-1-positive components \\
\hline $\mathrm{A}_{\mathrm{A}}($ granulocytes, thr $)$ & $40 \times$ & $1(8)$ & 1.59 & $>150$ intersections with granulocyte-positive areas \\
\hline $\mathrm{A}_{\mathrm{A}}($ macrophages, thr $)$ & $40 \times$ & $1(8)$ & 1.59 & $>150$ intersections with macrophage-positive areas \\
\hline
\end{tabular}

The microscope objective and magnification used for the quantitative assessment of each of the parameters was at the lowest setting that permitted an exact and unambiguous identification of the counting events with respect to histological staining methods. The number of counting events per sample is provided, and the resulting data are presented as arithmetic means calculated from stochastic methods. Quantification of all of the parameters in the aortic wall was based on a total of 6,072 micrographs,

and quantification of thrombi was based on 1,072 micrographs. $\mathrm{A}_{\mathrm{A}}$ (component, space): Area fractions of the respective components within their reference spaces; $\mathrm{L}_{\mathrm{A}}$ : two-dimensional length density of elastin fibres within the intima and media; $Q_{A}$ : number of microvessel profiles per section area; int+med: data pooled from the intima and media; wall: data pooled from the wall (i.e. from the intima, media and adventitia); thr: data pooled from the thrombus.

\section{Histological Quantification}

Quantitative analysis was based on stereological methods using Ellipse software (ViDiTo, Košice, Slovakia). All of the methods used in this study have recently been established, evaluated, and described in detail $[32,33$; for an explanation of the techniques, see 34]. Sampling of histological sections, microscopic image fields, definition of the reference area and the total area of the sections used for estimating the quantitative parameters are summarised in table 2 .

The principles of stereological estimations used in this study are shown in figure 1 . Briefly, elastinolysis within the aortic wall was assessed using two-dimensional length density $\mathrm{L}_{\mathrm{A}}$ (length per area, $\mathrm{mm}^{-1}$ ) of profiles of elastin fibres and the area fraction $\mathrm{A}_{\mathrm{A}}$ (area per area, dimensionless ratio) of elastin within the intima and media. Within the same reference space, the collagen content was quantified as the area fraction of collagen within the intima and media. Elastin and collagen areas were quantified in sections stained with Verhoeff's haematoxylin and green trichrome (fig. 1a). The presence of cells expressing actin, desmin and vimentin was quantified as area fractions $\mathrm{A}_{\mathrm{A}}$ of these filaments within the intima and media (an example is shown in fig. 1b). To quantify the intra-aortic microvascular bed, the vasa vasorum was quantified as the number of CD31-positive microvessel profiles per section area $\mathrm{Q}_{\mathrm{A}}$ of the vascular wall (fig. 1c). Infiltration of the vascular wall by neutrophils and macrophages, which are recognised as a major source of proteases in inflammatory AAA infiltrates [26, 35], was characterised as the area fraction of the profiles of immunohistochemically detected neutrophils and macrophages within the aortic wall and the thrombus (fig. 1d, f). Cells expressing the regulators of fibrinolysis [36] were quantified as the area fractions of PAI-1-, tPA- and uPA-positive cells within the aortic wall (an example is shown in fig. 1e). Stereological methods used in the study were based on counting intersections of the structures of interest with stereological grids or counting frames randomly superposed on the micrographs. During the histological evaluation, the observers had no knowledge of the biological status of the samples or the patient histories.

\section{In vitro Immunoassays}

Concentrations of the interleukins (IL)-6, 8 and 10, the matrix metalloproteinases (MMP)-1, 2, 7 and 9, the tissue inhibitors of matrix metalloproteinases (TIMP)-1 and 2, the E-selectin, the vascular cell adhesion molecule (VCAM)-1 and the intercellular cell adhesion molecule (ICAM)-1, the plasminogen activator inhibitor-1 (PAI)-1, the epidermal growth factor (EGF), the vascular endothelial growth factor (VEGF) and the osteoprotegerin (OPG) were measured in the wall tissue and thrombus homogenates. Approximately 50 -mg centrally cut pieces were used from frozen tissue and thrombus samples and homogenised in buffer supplemented with Nonidet-P40, protease inhibitor and phenylmethanesulfonyl fluoride using ceramic particles and a FastPrepBIO101 disrupter (Thermo Savant, Holbrook, N.Y., USA). Homogenates 
were aliquoted and stored at $-70^{\circ} \mathrm{C}$. No more than 1 freeze-thaw cycle was allowed before an analysis. Prior to multiplex analysis, the aliquots were centrifuged for $5 \mathrm{~min}$ at $10,000 \mathrm{~g}$ to remove any clots or particles. Measurements were performed by bead-based multi-analyte profiling technology (xMAP) using the Luminex 100 IS system (Luminex Corporation, Austin, Tex., USA) and the commercially available multiplex panels consisting of the human cytokine/chemokine Milliplex MAP kit, human cardiovascular disease panel (both from Millipore, Billerica, Mass., USA), and the fluorokine MAP human MMP kit (R\&D Systems, Minneapolis, Minn., USA).

xMAP technology is a multiplex immunoanalytic technology ligand binding assay that combines sandwich immunoassays and flow cytometry and enables quantification of up to 100 samples at once. It is based on the binding of target proteins to antibodies linked to microspheres with an internal spectral code that refers to the protein identity. The amount of bound protein is determined using a secondary antibody labelled with a fluorescent molecule. The measurement is performed using a flow cytometer that is capable of determining the spectral code of the microspheres or the identity of the proteins, after excitation by a laser. After excitation by a second laser, the flow cytometer detects the amount of secondary antibodies bound to the microspheres and quantifies the amount of protein in the sample. The concentrations of the proteins are assessed according to standard calibration curves that are assayed together with the unknown samples.

TIMP-1 and TIMP-2 levels were measured with ELISA technology using commercial kits (R\&D Systems, Vienna, Austria). The total amount of protein in the homogenates was quantified using a bicinchoninic acid assay (Bicinchonic Acid Protein Assay Kit, Sigma Aldrich, Mo., USA). The total protein concentration was used for data standardisation. All used commercial in vitro assays were tested by manufacturers for specificity. The antibody pairs in the panels were specific only to the desired analyte and exhibited no or negligible cross-reactivity with other analytes.

\section{Statistics}

The data were processed with Statistica Base 9 (StatSoft Inc., Tulsa, Okla., USA). The Shapiro-Wilks W test was used for normality testing. Because normality was rejected for most variables, we used the non-parametric Mann-Whitney U test for testing the equality of population medians between each pair of the groups under study (according to the biological condition of the sample, patient symptoms and AAA diameter). For paired samples of the AAA wall and adjacent thrombi we used the Wilcoxon matched pairs test. The Spearman rank order correlation was used to measure statistical relationships between continuous variables. Values were considered statistically significant for $\mathrm{p}<0.05$. Only significant findings are reported.

\section{Results}

\section{Extracellular Matrix and Regulators of Fibrinolysis}

The area fraction and the length density of elastin within the intima and media of the normal aorta were greater than in the AAAs (fig. 2a). In contrast, the area fraction of collagen in the same reference location was greater in the AAAs than in the normal aorta (fig. 2a). The area fraction of collagen within the same reference location of symptomatic AAAs was greater than in asymptomatic AAAs but did not reach statistical significance $(p=0.058)$. The area fraction of PAI-1 within the wall was greater in the normal aorta than in the AAAs (fig. 2a). The area fraction of PAI-1-positive tissue elements in the wall was greater in symptomatic than in asymptomatic AAAs (fig. 2e), while the area fraction of tPA-positive elements in the same reference location was greater in asymptomatic AAAs (fig. 2e) compared with symptomatic cases.

\section{Expression of Actin, Desmin and Vimentin}

Actin, desmin and vimentin were used to characterise the vascular smooth muscle cells of intima and media of the aortic wall. There was a higher degree of positive staining for actin and desmin within the intima and media in the normal aorta than in AAAs (fig. 2b). Mediumsized AAAs had a greater area fraction of both actin- and vimentin-positive cell profiles than the large AAAs (fig. 2d). Medium-sized AAAs had a greater area fraction of vimentin-positive elements within the intima and media than small AAAs (fig. 2d).

\section{Vasa Vasorum and Inflammatory Cells}

The number of microvessel profiles per section area was greater in the normal aorta than in AAAs (fig. 2b). The number of microvessel profiles increased with the diameter of the AAA (fig. 2d) and was greater in large AAAs than in small AAAs (fig. 2d). The area fraction of granulocytes and macrophages was greater in the AAA wall than in the adjacent thrombi (fig. 2c). The area fraction of granulocytes and macrophages was greater in asymptomatic AAAs than in symptomatic AAAs (fig. 2e).

\section{Wall Homogenates}

The immunoassay showed a greater concentration of VCAM-1 within the wall of small AAAs than in the wall of large AAAs (fig. 2f). The concentration of IL-8 was greater in the wall of large AAAs than in the wall of small AAAs (fig. 2f) and the concentration of VEGF was greater in the wall of large AAAs than in the wall of mediumsized AAAs (fig. 2f).

\section{Thrombi Homogenates}

The immunoassay showed a greater concentration of VCAM-1 and MMP-2 within the thrombi from asymp- 
tomatic AAAs than in the thrombi from symptomatic AAAs (fig. 2g). The concentration of MMP-9 was greater in the thrombi from ruptured AAAs than in the thrombi from asymptomatic AAAs (fig. 2g). The concentration of VCAM-1 was greater in the thrombi from small and large AAAs than in the thrombi from medium-sized AAAs (fig. $2 \mathrm{~h}$ ). The concentration of ICAM-1 was greater in the thrombi from large AAAs than in the thrombi from medium-sized AAAs (fig. 2h).

\section{Correlation of Quantitative Parameters}

The non-parametric Spearman rank order correlations between quantitative parameters are listed in table 3 , and the significant values $(\mathrm{p}<0.05)$ are highlighted.

\section{Discussion}

Some but not all quantitative parameters assessed in AAA samples varied in relation to severity of the disease, i.e. presence of clinical symptoms and AAA size (fig. $2 \mathrm{~d}-$ h). Similarly, differences between the wall of normal aortae and AAA as well as between AAA wall and thrombus could be found for some of the data (fig. 2a-c).

\section{Collagen, Elastin and Fibrinolysis}

Fragmentation and loss of elastic fibres accompanied by increased collagen content is the most striking histological feature of the aneurysmal wall when compared to the normal aorta. In contrast to these findings, our results did not confirm the generally accepted principles of AAA progression, in which the loss of elastin is an early step in aneurysm formation $[16,37]$ and collagen degradation is the ultimate cause of rupture [37]. We found a slightly higher collagen content in symptomatic AAAs when compared to asymptomatic. This confirms the results of Deguchi et al. [38] who reported an increased aortic collagen content to be linked with the AAA formation.

Degradation of fibres of the aortic wall is thought to be triggered by plasmin, either directly or via MMPs [39, 40]. This is confirmed by the low fraction of the PAI-1positive cells (PAI-1 is an inhibitor of fibrinolysis) in AAAs found in our study. Surprisingly, we detected higher levels of tPA and lower levels of PAI-I in asymptomatic AAAs (fig. 2e) when compared with AAAs causing clinical symptoms. This might indicate that especially smaller and clinically silent AAAs are prone to progression.

The positive correlation between tPA levels and the quantity of macrophages (inflammation) and vimentinpositive elements (synthetic VSMC phenotype) within the vessel wall and the negative correlation between tPA and collagen content (degradation of the vessel wall matrix) support this assumption. Interestingly, the fraction of UPA with pro-fibrinolytic activity was positively correlated with the contractile VSMC phenotype (actin- and desmin-positive cells), which suggests that fibrinolysis was not compensated by the presence of the synthetic VSMC phenotype.

\section{The Shift of the VSMC Phenotype during AAA \\ Development Weakens the Aortic Wall}

The shift of VSMC from actin- and desmin-expressing contractile to vimentin-expressing synthetic phenotype (fig. 2b) has been shown before [17, 41, 42]. We found most actin- and vimentin-positive elements in medium-sized

Fig. 1. Quantitative histopathology of aortic samples. a Estimating length density of elastin profiles (length per area of tunica media and intima) of an asymptomatic AAA sample. Intersections between the circular arcs (yellow) and elastin fibres (black) were counted (orange) and the length of elastin profiles was calculated according to the modified Buffon method [34]. b When estimating the area fraction of the detected structures, points hitting the profiles were counted (marked yellow), and their sum was multiplied by the area corresponding to each point (marked 'a' within the square). This is demonstrated for the quantification of the area of $\alpha$-smooth muscle actin within the intima and media of an AAA sample. The reference area was assessed in the same way, and the relation between area of actin-positive structures and reference area was calculated. c Counting the number of microvessel profiles per section area in the wall of an AAA using an unbiased counting frame [34]. Profiles of microvessels (green points) inside the frame or touching the green admittance borders but not touching the red forbidden lines were counted. $\mathbf{d}$ The presence of inflammatory cells was quantified as the area fraction occupied by the profiles of the cells per sectional area of the wall. This is demonstrated for macrophages that accumulated at the base of an atherosclerotic plaque within the tunica intima and media of an AAA sample. e The cells expressing regulators of fibrinolysis were quantified using their area fractions within the wall. This is demonstrated for various PAI-1-positive cells at the border between the intima and media of an AAA sample. Quantification was as described in $\mathbf{b}$. $\mathbf{f}$ Neutrophilic granulocytes in the adluminal layer of the thrombus (upper part of the micrograph) [25]. Verhoeff's haematoxylin and green trichrome staining (a) with elastin stained as black lamellae and fibres, collagen stained green to blue, and smooth muscle cells stained reddish to brown. $\alpha$-Smooth muscle actin (b), CD31-positive endothelial cells (c), the macrophage L1 protein calprotectin (d), PAI-1 (e), human granulocytic myeloperoxidase (f) were detected immunohistochemically, visualised with horseradish peroxidase/diaminobenzidine and counterstained with haematoxylin. Scale bar $=50 \mu \mathrm{m}$ (a, b, d-f) and $200 \mu \mathrm{m}(\mathbf{c})$. 

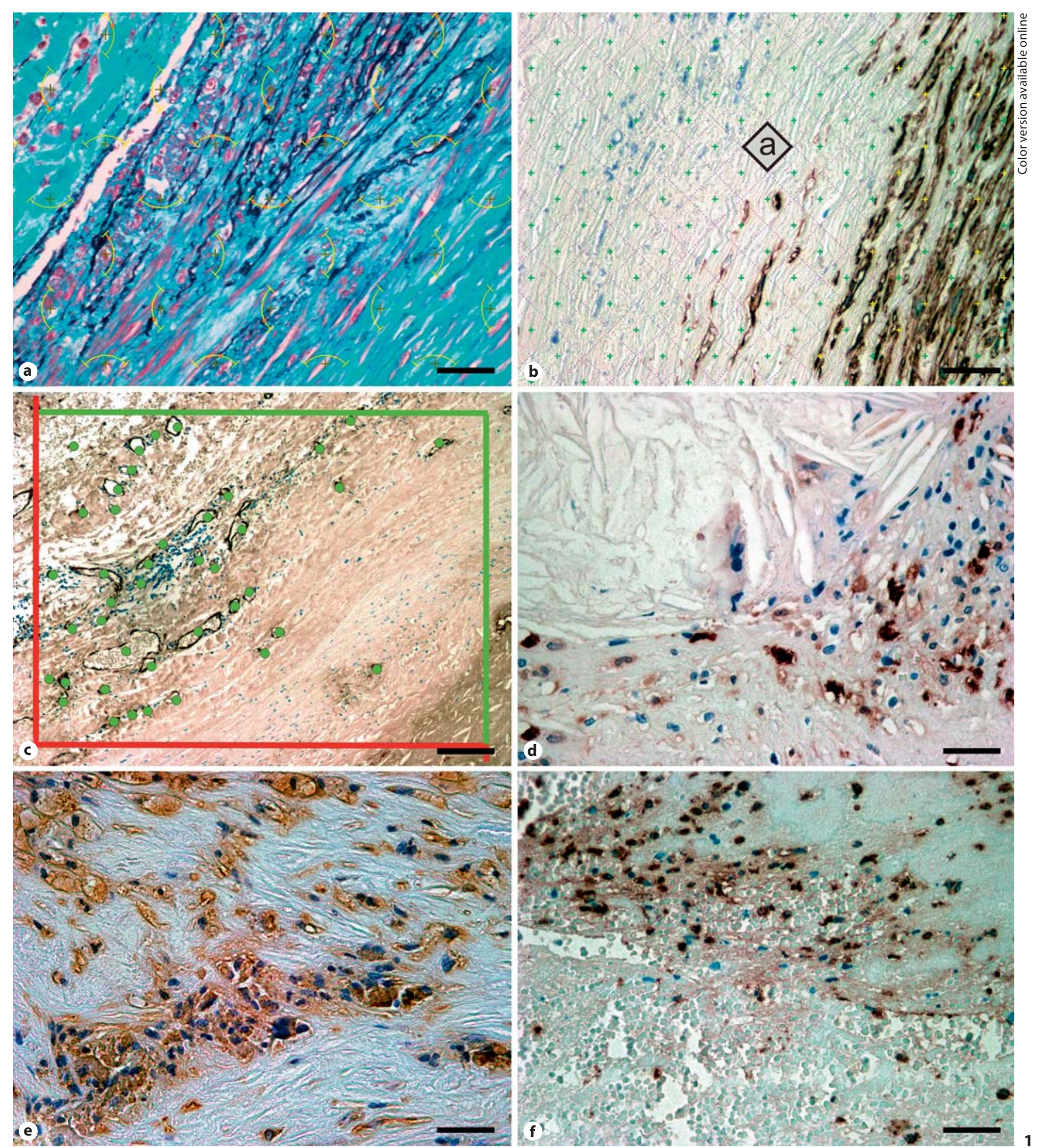

Asymptomatic Aortic Aneurysm - Risky

Pathobiology 2013;80:11-23 


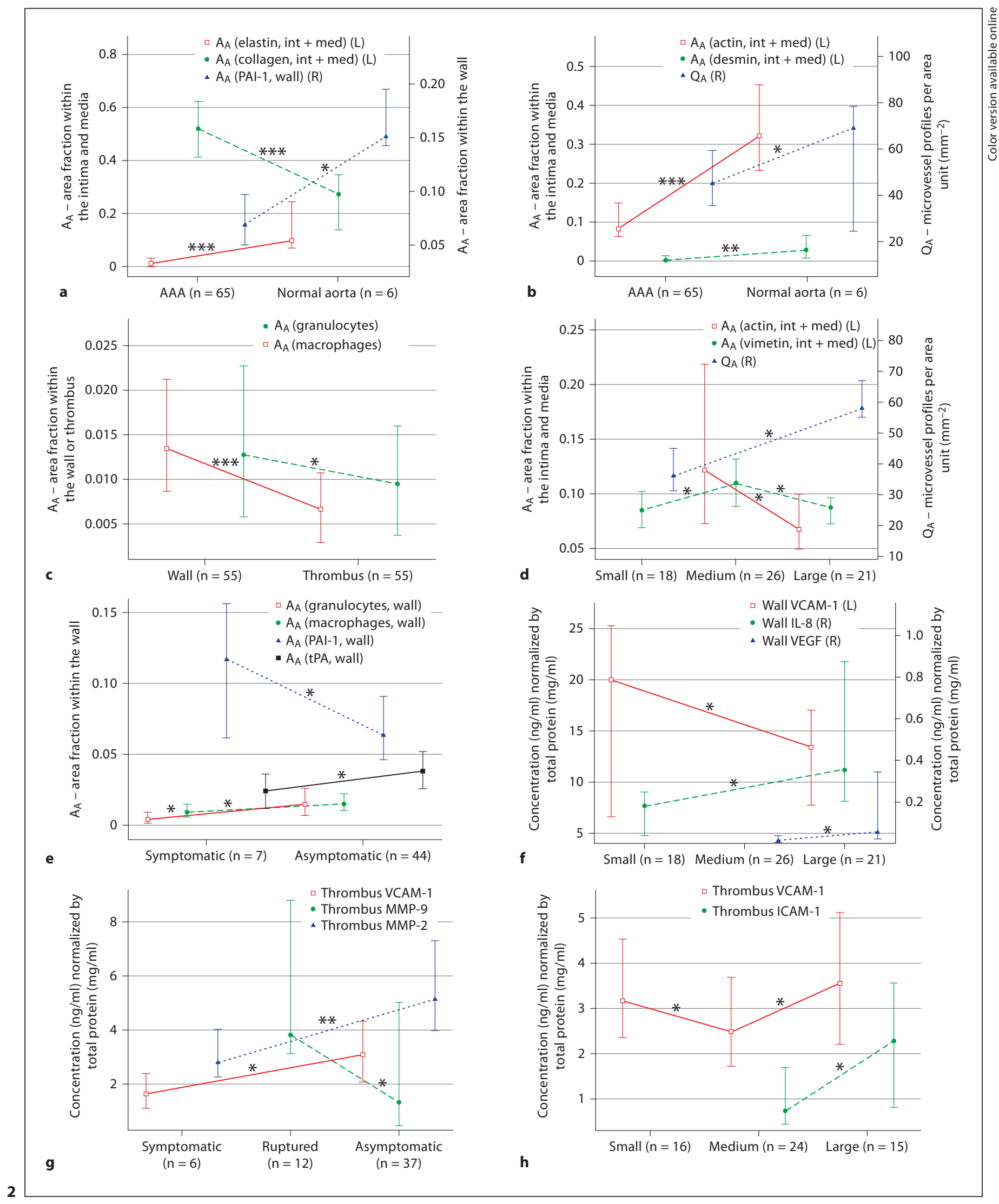


AAAs (fig. 2d), corroborating the findings of Ailawadi et al. [17] who observed VSMC phenotypic modulation and decreased $\alpha$-smooth muscle actin expression in early AA A development. Drop-offs in both actin and vimentin positivity in large AAAs are most likely caused by apoptosis of VSMCs $[41,18]$. Modulation of VSMC phenotype with loss of contractile cells and apoptotic cell death might represent important factors leading to the mechanical weakening of the AAA wall [43].

\section{A High Density of Vasa Vasorum in Large Aneurysms}

Complementing the findings of Acoltzin Vidal et al. [44], we have shown a significantly lower microvessel density in AAA samples (fig. 2b) which suggests an imbalance between wall dilatation and reparative adventitial angiogenesis [45]. A high density of vasa vasorum may occur in a healthy vascular wall, but it might also be due to hypoxia-driven neovascularization and inflammatory angiogenesis, thus finally exceeding metabolic requirements of the tissue [46]. Interestingly, in our material microvessel density increased with the AAA diameter (fig. $2 \mathrm{~d}$ ). This could be explained by the greater VEGF expression we found in large AAAs (fig. 2f). VEGF might be produced by the increased inflammatory infiltrates in the AAA wall, because the infiltrates are known to be correlated with increased aortic wall vascularity $[47,48]$. However, formation of new fragile vessels could be an additional factor enhancing the vulnerability of the vascular wall [49]. In accordance with another study [48], we showed a positive correlation between vessel wall vascularity and inflammatory infiltrates in AAA walls. In-

Fig. 2. Quantitative histopathology of the aortic walls and thrombi. $\mathbf{a}-\mathbf{g}$ The data are presented as medians and the whiskers span the interquartile range. The differences were considered statistically significant if ${ }^{*} p<0.05{ }^{* *} p<0.01$ and ${ }^{* * *} p<0.001$. a, b Area fractions of elastin, collagen, actin and desmin within the intima and media ( $\mathrm{L}=$ left axis); area fraction of PAI-1 in the wall; and the microvessel profiles per area unit within the wall ( $\mathrm{R}=$ right axis) when comparing the AAA with normal aortas. c Comparing the area fractions of granulocytes and macrophages within the AAA wall and within adjacent thrombi of the same patients. $\mathbf{d}$ The area fractions of actin and vimentin within the wall (left axis) and the microvessel profiles per area unit (right axis) in small, medium and large AAAs. e The area fractions of granulocytes, macrophages, PAI-1 and tPA within the wall in symptomatic and asymptomatic AAAs. $\mathbf{f}, \mathbf{g}, \mathbf{h}$ Concentration of proteins (VCAM-1, IL-8, VEGF, MMP-2, MMP-9, ICAM-1) assessed with an immunoassay within the homogenates of the AAA wall (f) and adjacent thrombi $(\mathbf{g}, \mathbf{h})$.

Asymptomatic Aortic Aneurysm - Risky and Rupture-Prone? creased microvessel density in large AAAs also corresponds to the greater concentration of IL-8 in large AAAs, because IL- 8 is a potent angiogenic factor and chemoattractant overexpressed at the AAA rupture side [50].

\section{Asymptomatic AAAs Are More Densely Infiltrated by Inflammatory Cells}

Increased transmural inflammation is associated with AAA progression [51]. The more pronounced inflammatory infiltration in asymptomatic AAA in comparison with symptomatic aneurysms was the most striking result of our study (fig. 2f). However, this finding supports the model of Yoshimura et al. [28], who distinguished a central region of large aneurysms with a thin, amorphous wall and thick, inactive thrombus from the neck region of aneurysms with thin thrombus, active inflammation and increased angiogenesis. Inflammatory cells produce proteolytic enzymes, including MMPs and elastase, that degrade the structural components of the aortic wall [52, $22,25]$. The positive correlation between the quantity of granulocytes in the wall and MMP-1 in thrombi, and the negative correlation between the wall granulocytes and TIMP-2 found in our material confirms this finding. Granulocyte infiltration in AAA walls and thrombi was positively correlated with the presence of IL- 6 and IL-8. The levels of these interleukins are known to be high in patients with (large) aneurysms [20,53].

\section{Thrombus and Inflammation}

The importance of immunological and inflammatory gene pathways in AAA pathogenesis was suggested by Lenk et al. [54], who reported overexpression of $M M P 1$, $M M P 9, I L 1 B$ and PLAUR (urokinase activator) genes. In the present study, MMP-9, considered to be a biomarker of AAA progression [55], was higher in thrombi from ruptured AAAs than in thrombi from asymptomatic AAAs.

Although macrophages are commonly considered to be a primary source of cytokines [20], we also found a correlation between granulocytes in the thrombus and the IL-8 in the same samples (table 3). This is in agreement with Houard et al. [26] who suggested the neutrophil-derived IL-8 to be involved in attracting neutrophils to the thrombus. Interestingly, the area fraction of granulocytes in both the wall and the thrombus correlated negatively with TIMP-2 levels in the aneurysmatic wall. In addition to MMP inhibition, TIMP-2 abrogates angiogenesis [56] by inhibiting the principal AAA pathogenic factors. The increased concentrations of VCAM-1 and MMP-2 in thrombi of asymptomatic AAA 


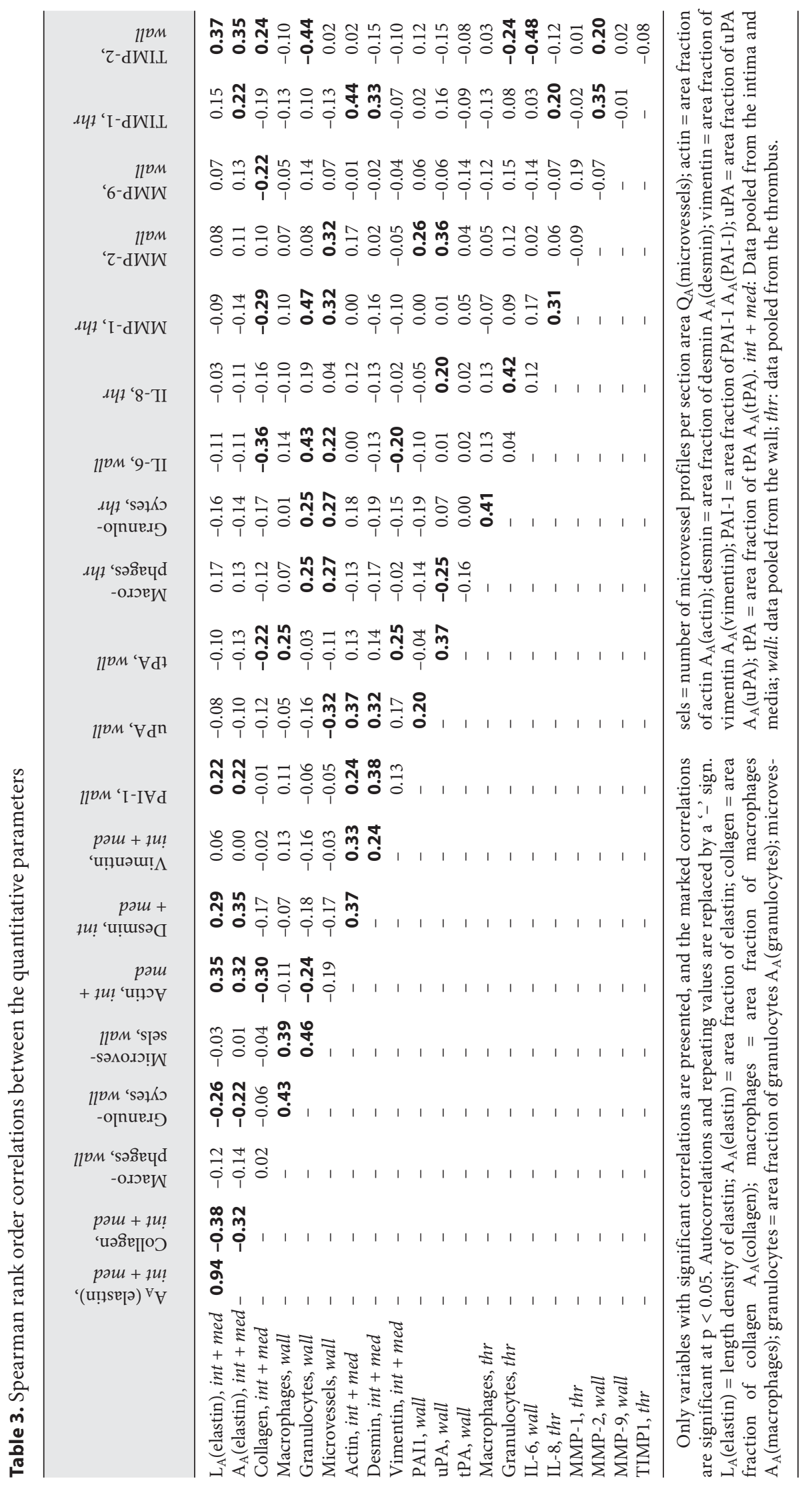


(fig. $2 \mathrm{~g}$ ) strongly support the rupture-prone nature of the asymptomatic AAAs indicated by the quantitative histopathology.

\section{Limitations of the Study}

We are aware of several limitations of our study. First, the number of AAA patients and the number of healthy control samples is relatively low and the controls do not match the AAA group in age, gender or comorbidities. The healthy aortas were collected from organ donors only to guarantee the same handling of tissue specimens as in AAA tissue samples excised during AAA surgery. Thus we prevented unequal or extended time intervals prior to the tissue fixation. Second, we were lacking the precise AAA diameter in emergency patients with AAA rupture since any delay prior to the surgery (such as CT) would threaten the patient's life. Thus, the diameter of AAA had to be classified into three categories (small, medium and large). These categories are artificial but are also used in clinical practice $[57,58]$. However, our study is lacking a detailed statistical analysis using the diameter as a continuous variable. Third, our quantification used two-dimensional routine sections only, whereas some structures (e.g. the vasa vasorum) are three-dimensional and would require more sophisticated quantification techniques, e.g. confocal microscopy or X-ray microtomography [46].

In conclusion, our results reveal the seriousness of asymptomatic AAAs. Although most of our asymptomatic patients were treated with statins or acetylsalicylic acid, both of which have an anti-inflammatory effect, the wall of the asymptomatic AAA exhibited more abundant inflammatory infiltrates, a greater area fraction of tPA, lower PAI-1 positivity in the same area fraction, lower collagen content in the intima and media, whereas the adjacent thrombi had a greater concentration of VCAM-1 and MMP-2 when compared to the symptomatic AAA. All of these findings are indicative of AAA progression. The microvessel density was greater in normal aorta samples and appears to participate in AAA progression because it increases with the size of the AAA. The intima and media of the medium-sized AAAs with diameters of 5-7 cm were richest in actin and vimentin. Surprisingly, the content of elastin and collagen did not significantly change with the size of the AAA. The normal aorta contains more elastin and VSMCs with the contractile phenotype and more PAI-1-positive elements.

The diagnosis of small asymptomatic AAAs is usually incidental in the absence of routine screening. Because most AAAs remain asymptomatic (even in the case of progression), our results strongly support the need for AAA screening programs, which have recently been implemented in the USA, the UK and Sweden, to detect small aneurysms with preventable progression.

\section{Acknowledgements}

We wish to thank Ms. B. Machac, Ms. E. Kielhausen and Ms. M. Helmreich for their excellent technical assistance. This work was supported by the European Union under the 7th FP of the EU project Fighting Aneurysmal Disease, No. 200647, and by the Ministry of Education, Youth and Sports of the Czech Republic under the research project MSM 0021620819. Quantification of microvessels was funded by the Charles University in Prague, project No. SVV 264808. This work was partially supported by the European Regional Development Fund (ERDF) project 'NTIS - New Technologies for Information Society', European Centre of Excellence, CZ.1.05/1.1.00/02.0090.

\section{References}

1 Michel JB, Martin-Ventura JL, Egido J, Sakalihasan N, Treska V, Lindholt J, Allaire E, Thorsteinsdottir U, Cockerill G, Swedenborg J; FAD EU consortium: Novel aspects of the pathogenesis of aneurysms of the abdominal aorta in humans. Cardiovasc Res 2011;90:18-27.

\2 Wassef M, Upchurch GR Jr, Kuivaniemi H, Thompson RW, Tilson MD 3rd: Challenges and opportunities in abdominal aortic aneurysm research. J Vasc Surg 2007;45:192-198.

$\checkmark 3$ Johnston KW, Rutherford RB, Tilson MD, Shah DM, Hollier L, Stanley JC, et al: Suggested standards for reporting on arterial aneurysms. J Vasc Surg 1991;13:452-458.
4 Bickerstaff LK, Hollier LH, Van Peenen HJ, Melton LJ 3rd, Pairolero PC, Cherry KJ: Abdominal aortic aneurysms: the changing natural history. J Vasc Surg 1984;1:6-12.

5 Darling RC, Messina CR, Brewster DC, Ottinger LW: Autopsy study of unoperated abdominal aortic aneurysms: the case for early resection. Circulation 1977;56(suppl. 3):161164.

6 Heikkinen M, Salenius JP, Auvinen O: Ruptured abdominal aortic aneurysm in a welldefined geographic area. J Vasc Surg 2002; 36:291-296.
7 Lederle FA, Johnson GR, Wilson SE, et al: Rupture rate of large abdominal aortic aneurysms in patients refusing or unfit for elective repair. JAMA 2002;287:2968-2972.

$>8$ Lindholt JS, Juul S, Fasting H, Henneberg EW: Screening for abdominal aortic aneurysms: single centre randomised controlled trial. BMJ 2005;330:750.

$\checkmark 9$ Norman PE, Jamrozik K, Lawrence-Brown MM, Le MT, Spencer CA, Tuohy RJ, Parsons RW, Dickinson JA: Population based randomised controlled trial on impact of screening on mortality from abdominal aortic aneurysm. BMJ 2004;329:1259. 
10 Eickhoff JH: Incidence of diagnosis, operation and death from abdominal aortic aneurysms in Danish hospitals: results from a nation-wide survey, 1977-1990. Eur J Surg 1993; 159:619-623.

-11 Filipovic M, Goldacre MJ, Roberts SE, Yeates D, Duncan ME, Cook-Mozaffari P: Trends in mortality and hospital admission rates for abdominal aortic aneurysm in England and Wales. 1979-1999. Br J Surg 2005;92:968975.

-12 Svensjö S, Björck M, Gürtelschmid M, Djavani Gidlund K, Hellberg A, Wanhainen A: Low prevalence of abdominal aortic aneurysm among 65-year-old Swedish men indicates a change in the epidemiology of the disease. Circulation 2011;124:1118-1123.

-13 Sandiford P, Mosquera D, Bramley D: Trends in incidence and mortality from abdominal aortic aneurysm in New Zealand. Br J Surg 2011;98:645-651

-14 Treska V, Kocova J, Boudova L, Neprasova P, Topolcan O, Pecen L, Tonar Z: Inflammation in the wall of abdominal aortic aneurysm and its role in the symptomatology of aneurysm. Cytokines Cell Mol Ther 2002;7:9197.

-15 Holmes DR, Liao S, Parks WC, Thompson RW: Medial neovascularization in abdominal aortic aneurysms: a histopathologic marker of aneurysmal degeneration with pathophysiologic implications. J Vasc Surg 1995;21:761-771.

-16 Sakalihasan N, Heyeres A, Nusgens BV, Limet R, Lapière CM: Modifications of the extracellular matrix of aneurysmal abdominal aortas as a function of their size. Eur J Vasc Surg 1993;7:633-637.

$\checkmark 17$ Ailawadi G, Moehle CW, Pei H, Walton SP, Yang Z, Kron IL, Lau CL, Owens GK: Smooth muscle phenotypic modulation is an early event in aortic aneurysms. J Thorac Cardiovasc Surg 2009;138:1392-1399.

- 18 Henderson EL, Geng YJ, Sukhova GK, Whittemore AD, Knox J, Libby P: Death of smooth muscle cells and expression of mediators of apoptosis by $\mathrm{T}$ lymphocytes in human abdominal aortic aneurysms. Circulation 1999;99:96-104.

$>19$ Lindholt JS, Shi GP: Chronic inflammation, immune response, and infection in abdominal aortic aneurysms. Eur J Vasc Endovasc Surg 2006;31:453-463.

-20 Sprague AH, Khalil RA: Inflammatory cytokines in vascular dysfunction and vascular disease. Biochem Pharmacol 2009;78:539_ 552 .

-21 Choke E, Cockerill G, Wilson WR, Sayed S, Dawson J, Loftus I, Thompson MM: A review of biological factors implicated in abdominal aortic aneurysm rupture. Eur J Vasc Endovasc Surg 2005;30:227-244.

-22 Thompson RW, Parks WC: Role of matrix metalloproteinases in abdominal aortic aneurysms. Ann NY Acad Sci 1996;800:157174.
23 Wolf YG, Thomas WS, Brennan FJ, Goff WG, Sise MJ, Bernstein EF: Computed tomography scanning findings associated with rapid expansion of abdominal aortic aneurysms. J Vasc Surg 1994;20:529-535.

24 Vorp DA, Lee PC, Wang DH, Makaroun MS, Nemoto EM, Ogawa S, Webster MW: Association of intraluminal thrombus in abdominal aortic aneurysm with local hypoxia and wall weakening. J Vasc Surg 2001;34:291-299.

25 Fontaine V, Jacob MP, Houard X, Rossignol P, Plissonnier D, Angles-Cano E, Michel JB: Involvement of the mural thrombus as a site of protease release and activation in human aortic aneurysms. Am J Pathol 2002;161: 1701-1710.

-26 Houard X, Ollivier V, Louedec L, Michel JB, Bäck M: Differential inflammatory activity across human abdominal aortic aneurysms reveals neutrophil-derived leukotriene B4 as a major chemotactic factor released from the intraluminal thrombus. FASEB J 2009;23. 1376-1383.

27 Doyle BJ, Callanan A, McGloughlin TM: A comparison of modelling techniques for computing wall stress in abdominal aortic aneurysms. Biomed Eng Online 2007;6:38.

28 Yoshimura K, Ikeda Y, Aoki H: Innocent bystander? Intraluminal thrombus in abdominal aortic aneurysm Atherosclerosis 2011; 218:285-286.

29 Lindholt JS: Abdominal aortic aneurysms. Dan Med Bull 2010;57:B4219.

30 Cooper DG, King JA, Earnshaw JJ: Role of medical intervention in slowing the growth of small abdominal aortic aneurysms. Postgrad Med J 2009;85:688-692.

31 Golledge J, Norman PE: Current status of medical management for abdominal aortic aneurysm. Atherosclerosis 2011;217:57-63.

32 Witter K, Tonar Z, Matejka VM, Martinca T, Jonák M, Rokosný S, Pirk J: Tissue reaction to three different types of tissue glues in an experimental aorta dissection model: a quantitative approach. Histochem Cell Biol 2010;133:241-259.

33 Tonar Z, Witter K, Křížková V, Eberlová L, Kočová J, Moláček J, Houdek K, Kochová $\mathrm{P}$, Vrzalová J, Topolčan O, Třeška V: Stereological tools for quantitative microscopy of the aortic wall with focus on the abdominal aortic aneurysm; in Méndez-Vilas A, Díaz J (ed): Microscopy: Science, Technology, Applications and Education - Applications in Biology and Medicine. Badajoz, Formatex Research Centre, 2010, pp 926-935.

34 Howard CV, Reed MG: Unbiased Stereology: Three-Dimensional Measurement in $\mathrm{Mi}$ croscopy, ed 1. New York, Royal Microscopical Society, 1998.

35 Houard X, Touat Z, Ollivier V, Louedec L, Philippe M, Sebbag U, Meilhac O, Rossignol P, Michel JB: Mediators of neutrophil recruitment in human abdominal aortic aneurysms. Cardiovasc Res 2009;82:532-541.
36 Houard X, Rouzet F, Touat Z, Philippe M, Dominguez M, Fontaine V, Sarda-Mantel L, Meulemans A, Le Guludec D, Meilhac O, Michel JB: Topology of the fibrinolytic system within the mural thrombus of human abdominal aortic aneurysms. J Pathol 2007; 212:20-28.

37 Dobrin PB, Mrkvicka R: Failure of elastin or collagen as possible critical connective tissue alterations underlying aneurysmal dilatation. Cardiovasc Surg 1994;2:484-488.

$\checkmark 38$ Deguchi JO, Huang H, Libby P, Aikawa E, Whittaker P, Sylvan J, Lee RT, Aikawa M: Genetically engineered resistance for MMP collagenases promotes abdominal aortic aneurysm formation in mice infused with angiotensin II. Lab Invest 2009;89:315-326.

39 Lijnen HR: Plasmin and matrix metalloproteinases in vascular remodeling. Thromb Haemost 2001;86:324-333.

40 Carmeliet P, Moons L, Lijnen R, Baes M, Lemaître V, Tipping P, Drew A, Eeckhout Y, Shapiro S, Lupu F, Collen D: Urokinase-generated plasmin activates matrix metalloproteinases during aneurysm formation. Nat Genet 1997;17:439-444

41 López-Candales A, Holmes DR, Liao S, Scott MJ, Wickline SA, Thompson RW: Decreased vascular smooth muscle cell density in medial degeneration of human abdominal aortic aneurysms. Am J Pathol 1997;150: 993-1007.

42 Tanasković I, Mladenović-Mihailović A, Usaj-Knezević S, Stanković V, Aleksić A, Kastratović T, Aleksić A, Lazić Z, Mladenović-Bogdanović Z, Zivanović A, Djurić J, Jovicić U, Sorak M: Histochemical and immunohistochemical analysis of ruptured atherosclerotic abdominal aortic aneurysm wall (in Serbian). Vojnosanit Pregl 2010;67:959-964.

43 Miyazaki H, Hayashi K, Hasegawa Y: Tensile properties of fibroblasts and vascular smooth muscle cells. Biorheology 2003;40: 207-212.

44 Acoltzin Vidal C, Maldonado Villaseñor I, Rodríguez Cisneros L, Muñiz Murguía JJ: Diminished vascular density in the aortic wall: morphological and functional characteristics of atherosclerosis. Arch Cardiol Mex 2004;74:176-180.

-45 Zollikofer CL, Redha FH, Bruhlmann WF, Uhlschmid GK, Vlodaver Z, CastanedaZuniga WR, Amplatz K: Acute and longterm effects of massive balloon dilation on the aortic wall and vasa vasorum. Radiology 1987;164:145-149.

46 Mulligan-Kehoe MJ: The vasa vasorum in diseased and nondiseased arteries. Am J Physiol Heart Circ. Physiol 2010;298:H295H305.

47 Paik DC, Fu C, Bhattacharya J, Tilson MD: Ongoing angiogenesis in blood vessels of the abdominal aortic aneurysm. Exp Mol Med 2004;36:524-533. 
-48 Reeps C, Pelisek J, Seidl S, Schuster T, Zimmermann A, Kuehnl A, Eckstein HH: Inflammatory infiltrates and neovessels are relevant sources of MMPs in abdominal aortic aneurysm wall. Pathobiology 2009;76: 243-252.

49 McCarthy MJ, Loftus IM, Thompson MM, Jones L, London NJ, Bell PR, Naylor AR, Brindle NP: Vascular surgical society of Great Britain and Ireland: angiogenesis and the atherosclerotic carotid plaque: association between symptomatology and plaque morphology. Br J Surg 1999;86:707-708.

50 Choke E, Cockerill GW, Laing K, Dawson J, Wilson WR, Loftus IM, Thompson MM: Whole genome-expression profiling reveals a role for immune and inflammatory response in abdominal aortic aneurysm rupture. Eur J Vasc Endovasc Surg 2009;37:305310 .
51 Freestone T, Turner RJ, Coady A, Higman DJ, Greenhalgh RM, Powell JT: Inflammation and matrix metalloproteinases in the enlarging abdominal aortic aneurysm. Arterioscler Thromb Vasc Biol 1995;15:11451151.

52 Coutard M, Touat Z, Houard X, Leclercq A, Michel JB: Thrombus versus wall biological activities in experimental aortic aneurysms. J Vasc Res 2010;47:355-366.

53 Lindholm E, Seljeflot I, Aune E, Kirkebøen KA: Proinflammatory cytokines and complement activation in salvaged blood from abdominal aortic aneurysm surgery and total hip replacement surgery. Transfusion 2012, E-pub ahead of print.

54 Lenk GM, Tromp G, Weinsheimer S, Gatalica Z, Berguer R, Kuivaniemi H: Whole genome expression profiling reveals a significant role for immune function in human abdominal aortic aneurysms. BMC Genomics 2007;8:237.
55 Lindholt JS, Vammen S, Fasting H, Henneberg EW, Heickendorff L: The plasma level of matrix metalloproteinase 9 may predict the natural history of small abdominal aortic aneurysms: a preliminary study. Eur J Vasc Endovasc Surg 2000;20:281-285.

56 Seo DW, Li H, Guedez L, Wingfield PT, Diaz T, Salloum R, Wei BY, Stetler-Stevenson WG: TIMP-2 mediated inhibition of angiogenesis: an MMP-independent mechanism. Cell 2003;114:171-180.

57 Paraskevas KI, Mikhailidis DP, Veith FJ: The rationale for lowering the size threshold in elective endovascular repair of abdominal aortic aneurysm. J Endovasc Ther 2011;18: 308-313.

58 Powell JT, Gotensparre SM, Sweeting MJ, Brown LC, Fowkes FG, Thompson SG: Rupture rates of small abdominal aortic aneurysms: a systematic review of the literature. Eur J Vasc Endovasc Surg 2011;41:2-10. 